\title{
ON THE ZETA FUNCTION OF BIPROJECTIVE COMPLETE INTERSECTIONS
}

\author{
BY \\ JACOB BARSHAY $\left({ }^{1}\right)$
}

Introduction. Let $p$ be a rational prime, $Q_{p}$ the field of $p$-adic numbers, $\Omega$ the completion of the algebraic closure of $Q_{p}$, and $k$ the finite field with $q=p^{a}$ elements. Denote by $k_{s}$ the extension field of $k$ of degree $s$ and $K$ the algebraic closure of $k_{s}$. By means of Teichmüller representatives, one can represent $K$ multiplicatively in $\Omega$. Specifically, the set of $\left(q^{s}-1\right)$-roots of unity in $\Omega$ represent the nonzero elements of $k_{s}$. Let $T_{s}$ be this set of Teichmüller representatives of $k_{s}$. Suppose now that $V$ is an irreducible nonsingular $\Omega$-projective variety defined over $Q_{p}\left(T_{1}\right)$ which gives rise by reduction $\bmod p$ to $\bar{V}$, an irreducible nonsingular $K$-projective variety defined over $k$. Denote by $N_{s}$ the number of points of $\bar{V}$ rational over $k_{s}$. Then the zeta function is defined to be

$$
\zeta(\bar{V}, t)=\exp \left(\sum_{s=1}^{\infty} N_{s} t^{s} / s\right)
$$

Dwork [3] has shown this to be a rational function of $t$ and it has been further conjectured by Weil [8] that

$$
\zeta(\bar{V}, t)=\prod_{j=0}^{2 d} P_{j}(t)^{(-1)^{j+1}}
$$

where $d=\operatorname{dim} V, P_{j}(t) \in Z[t], \operatorname{deg} P_{j}(t)=B_{j}=j$ th Betti number of $V$ for $j=0,1$, $\ldots, 2 d$. The conjecture has been verified for hypersurfaces by Dwork [4] and a weaker form (in a sense to be explained shortly) of the conjecture has been verified by Ireland [5] for projective complete intersections and biprojective divisors. It is these results of Ireland which are here extended to biprojective complete intersections. Throughout the paper we consider only intersections of two divisors but it will be clear that this is not an essential restriction and all results hold for arbitrary codimension.

Now a brief outline of the methods and results:

(1) A power series $F \in \Omega\{Z, X, Y\}$ is defined in terms of a splitting function $\theta(t)$ and the divisors $f$ and $g$. We then consider the endomorphism $\alpha=\psi \circ F$ of $\Omega\{Z, X, Y\}$ where $F$ stands for multiplication by $F$ and $\psi$ is the Dwork operator. We set $\chi_{F}(t)=\operatorname{det}(I-t \alpha)$ and obtain a formula of the form

$$
\chi_{F}^{\delta n+m+2}(t)=(1-t)^{G(n, m)} \prod_{A, C} P_{A, C}
$$

Received by the editors September 17, 1967.

(1) This paper constitutes part of the author's doctoral dissertation at Brandeis University. The author wishes to express his gratitude to K. F. Ireland for his advice and encouragement. 
where $\chi_{F}^{\delta}(t)=\chi_{F}(t) / \chi_{F}(q t), G(n, m)$ is some binomial function of the rational integers $n$ and $m\left(P^{n} \times P^{m}\right.$ is the biprojective space in which we are operating), and the $P_{A, C}$ are rational functions defined in terms of the zeta functions of all the varieties obtained from either of the two divisors or from their intersection by generic cuts.

(2) $\chi_{F}^{\delta^{n+m+2}}(t)$ is a polynomial. More specifically, $\chi_{F}^{\delta^{n+m+2}}(t)=\operatorname{det}(I-t \bar{\alpha})$ where $\bar{\alpha}$ is a nonsingular endomorphism of a quotient space of a space of power series whose coefficients satisfy certain growth conditions.

(3) The space on which $\bar{\alpha}$ operates is isomorphic to a finite-dimensional vector space $W$ whose dimension is computed via a Koszul complex resolution. The acyclicity of this complex follows from certain ring-theoretical considerations.

(4) The weaker form of the conjecture verified by Ireland and extended here can be stated as

$$
\zeta(\bar{V}, t)=\prod_{j=0}^{2 d} P_{j}(t)^{(-1)^{j+1}}
$$

where $d=\operatorname{dim} V$, for $j=0,1, \ldots, \hat{d}, \ldots, 2 d, P_{j}(t) \in Z[t], \operatorname{deg} P_{j}(t)=B_{j}$, and $P_{d}(t)$ $\in Z(t)$ with the degree of $P_{d}(t)$ as a rational function equal to $B_{d}$, the $d$ th Betti number of $V$. In all attempts thus far to extend Dwork's methods beyond hypersurfaces, no way has been found to isolate $P_{d}(t)$ and show it is a polynomial. More specifically, no generalization has been found for [4, Theorem 4.4].

The characteristic series $\chi_{F}$. Let $f(X, Y)=\sum_{i=1}^{r_{1}} A_{i} M_{i}$ and $g(X, Y)=\sum_{i=1}^{r_{2}} B_{i} M_{i}^{\prime}$ be bihomogeneous forms in $\Omega\left[X_{1}, \ldots, X_{n+1}, Y_{1}, \ldots, Y_{m+1}\right]=\Omega[X, Y]$ of bidegrees $\left(d_{1}, e_{1}\right)$ and $\left(d_{2}, e_{2}\right)$ respectively. Further assume that $A_{i}^{q}=A_{i}, B_{i}^{q}=B_{i}$. Denote by $\bar{V}$ the variety determined in $\boldsymbol{P}^{n}(K) \times \boldsymbol{P}^{m}(K)$ by the forms $\bar{f}(X, Y), \bar{g}(X, Y)$. We note that $\boldsymbol{P}^{n}(K) \times \boldsymbol{P}^{m}(K)$ can be embedded under the Segre map as a subvariety of $P^{(n+1)(m+1)-1}(K)$ of dimension $n+m$. We will assume that $\bar{V}$ is a nonsingular complete intersection, i.e. the dimension of $\bar{V}$ under this embedding is $n+m-2$.

Let $N_{s}$ be the number of points on $\bar{V}$ with no coordinate zero and rational over $k_{s}$. Let $\mathscr{H}_{s}(t)$ be the nontrivial $\Omega$-valued character of $k_{s}$ defined by Dwork [3]. Introducing new variables $Z_{1}, Z_{2}$ we obtain (where $k_{s}^{*}=k_{s}-\{0\}$ ):

$$
\begin{aligned}
q^{2 s}\left(q^{s}-1\right)^{2} N_{s}= & \sum_{z_{1}, z_{2} \in k_{s} ; x, y \in k_{s}^{*}} \mathscr{H}_{s}\left(z_{1} \bar{f}(x, y)+z_{2} \bar{g}(x, y)\right) \\
= & \left(q^{s}-1\right)^{n+m+2}+\sum_{z_{1}, x, y \in k_{s}^{*}} \mathscr{H}_{s}\left(z_{1} \bar{f}(x, y)\right)+\sum_{z_{2}, x, y \in k_{s}^{*}} \mathscr{H}_{s}\left(z_{2} \bar{g}(x, y)\right) \\
& +\sum_{z_{1}, z_{2}, x, y \in k_{s}^{*}} \mathscr{H}_{s}\left(z_{1} \bar{f}(x, y)+z_{2} \bar{g}(x, y)\right) .
\end{aligned}
$$

This formula follows from the fact that an $(n+m+2)$-tuple $\left(x_{1}, \ldots, x_{n+1}, y_{1}, \ldots\right.$, $y_{m+1}$ ) which represents a point of $\bar{V}$ contributes $q^{2 s}$ to the sum, whereas it contributes zero if it is not on $\bar{V}$. Also we must count points biprojectively. 
Now let $\theta(t)$ be any splitting function as defined by Dwork [4]. Define the following power series over $\Omega$ :

$$
\begin{aligned}
F_{1}\left(Z_{1}, X, Y\right) & =\prod_{i=1}^{r_{1}} \prod_{j=0}^{a-1} \theta\left(\left(Z_{1} A_{i} M_{i}\right)^{p^{\jmath}}\right), \\
F_{2}\left(Z_{2}, X, Y\right) & =\prod_{i=1}^{r_{2}} \prod_{j=0}^{a-1} \theta\left(\left(Z_{2} B_{i} M_{i}\right)^{p^{j}}\right), \\
F(Z, X, Y) & =F_{1}\left(Z_{1}, X, Y\right) \cdot F_{2}\left(Z_{2}, X, Y\right) .
\end{aligned}
$$

We recall the definition of the Dwork operator $\psi$. For any monomial

$$
\begin{array}{rlrl}
Z_{1}^{w} Z_{2}^{w_{2}} X_{1}^{u} \cdots X_{n+1}^{u_{n}+1} Y_{1}^{v} \cdots Y_{m+1}^{v_{m+1}}=Z^{w} X^{u} Y^{z}, & \\
\psi\left(Z^{w} X^{u} Y^{v}\right) & =Z^{w / q} X^{u / q} Y^{v / q} & & \text { if } q \text { divides every exponent, } \\
& =0 & & \text { otherwise. }
\end{array}
$$

Following [3, Lemma 2], we see that

$$
\begin{aligned}
q^{2 s}\left(q^{s}-1\right) N_{s}= & \left(q^{s}-1\right)^{n+m+2}+\left(q^{s}-1\right)^{n+m+3} \operatorname{Tr}\left(\psi \circ F_{1}\right)^{s} \\
& +\left(q^{s}-1\right)^{n+m+3} \operatorname{Tr}\left(\psi \circ F_{2}\right)^{s}+\left(q^{s}-1\right)^{n+m+4} \operatorname{Tr}(\psi \circ F)^{s}, \\
q^{2 s} N_{s}= & \left(q^{s}-1\right)^{n+m}+\left(q^{s}-1\right)^{n+m+1} \operatorname{Tr}\left(\psi \circ F_{1}\right)^{s} \\
& +\left(q^{s}-1\right)^{n+m+1} \operatorname{Tr}\left(\psi \circ F_{2}\right)^{s}+\left(q^{s}-1\right)^{n+m+2} \operatorname{Tr}(\psi \circ F)^{s} .
\end{aligned}
$$

Denote by $\bar{V}-\bar{V}^{\prime}$ the subset of $\bar{V}$ consisting of those points with no coordinate zero. Let $\delta$ be the operator $h(t)^{\delta}=h(t) / h(q t)$. Let $\chi_{F_{1}}(t)=\operatorname{det}\left(I-t\left(\psi \circ F_{1}\right)\right)$, $\chi_{F_{2}}(t)=\operatorname{det}\left(I-t\left(\psi \circ F_{2}\right)\right), \chi_{F}(t)=\operatorname{det}(I-t(\psi \circ F))$. Each of these is an entire function on $\Omega$.

Finally we obtain the following formula:

(1) $\zeta\left(\bar{V}-\bar{V}^{\prime}, q^{2} t\right)=(1-t)^{-(-\delta)^{n+m}} \chi_{F_{1}}^{-(-\delta)^{n+m+1}}(t) \chi_{F_{2}}^{-(-\delta)^{n+m+1}}(t) \chi_{F}^{-(-\delta)^{n+m+2}}(t)$.

Next we define the integers $B_{0}, B_{2}, B_{4}, \ldots, B_{2(n+m)}$ by

and set

$$
\left(\sum_{i=0}^{n} x^{i}\right)\left(\sum_{j=0}^{m} x^{j}\right)=\sum_{k=0}^{n+m} B_{2 k} x^{k}
$$

$$
\begin{array}{rlrl}
B_{2 j}(V) & =B_{2 j} & & \text { for } 2 j<n+m-2, \\
B_{2 j}(V) & =B_{2(n+m-2)-2 j} & & \text { for } 2(n+m-1)>2 j>n+m-2, \\
B_{2(n+m-1)}(V) & =B_{2(n+m)}(V)=1 . &
\end{array}
$$

Finally we define a rational function $P(t)$ by

where

$$
\zeta(\bar{V}, t)=\frac{P(t)^{(-1)^{n+m-1}}\left(1-q^{n+m-1} t\right)\left(1-q^{n+m} t\right)}{(1-t)^{N}},
$$

$$
\begin{aligned}
N & =\sum_{j=0}^{n+m} B_{2 j}(V) \phi^{j} \quad \text { if } n+m \text { is odd, } \\
& =\sum_{j=0 ; j \neq(n+m-2) / 2}^{n+m} B_{2 j}(V) \phi^{j}+B_{n+m-2} \phi^{(n+m-2) / 2} \text { if } n+m \text { is even. }
\end{aligned}
$$

Also $\phi$ denotes the operator $h(t)^{\phi}=h(q t)$. Note that $\delta=1-\phi$. 
The basis for these conventions is the Lefschetz theorem which states that the Betti numbers of $V$ agree with those of the ambient space except possibly for $B_{n+m-2}(V)$. Note that when $n+m-2$ is even, $P(t)$ is not the "Weil polynomial" but differs from it in degree by $B_{n+m-2}$ of $P^{n} \times P^{m}$.

We now proceed to generalize the above. Let $S_{1}=\left\{X_{1}, \ldots, X_{n+1}\right\}, S_{2}=$ $\left\{Y_{1}, \ldots, Y_{m+1}\right\}$. Suppose $A_{1} \subset S_{1}, A_{2} \subset S_{2}$, nonempty. Let $A=A_{1} \times A_{2}, 1+m\left(A_{i}\right)$ = number of elements in $A_{i}, m(A)=m\left(A_{1}\right)+m\left(A_{2}\right)$. For each subset $A$ of $S_{1} \times S_{2}$, consider the variety $V_{A}$ (resp. $\left.\bar{V}_{A}\right)$ in $P^{m\left(A_{1}\right)} \times P^{m\left(A_{2}\right)}$ (resp. $\left.P^{m\left(A_{1}\right)}(K) \times P^{m\left(A_{2}\right)}(K)\right)$ obtained by equating to zero in $f(X, Y)$ and $g(X, Y)$ (resp. $\bar{f}(X, Y)$ and $\bar{g}(X, Y)$ ) all those variables not in $A$. We assume that all of the resulting varieties are nonsingular.

If $m(A) \geqq 2$, we define a rational function $P_{A}(t)$ by

$$
\zeta\left(\bar{V}_{A}, t\right)=\frac{P_{A}(t)^{(-1)^{m(A)-1}}\left(1-q^{m(A)-1} t\right)\left(1-q^{m(A)} t\right)}{(1-t)^{N(A)}},
$$

where

$$
\begin{aligned}
N(A) & =\sum_{j=0}^{m(A)} B_{2 j}\left(V_{A}\right) \phi^{j} \quad \text { if } m(A) \text { is odd } \\
& =\sum_{j=0 ; j \neq(m(A)-2) / 2}^{m(A)} B_{2 j}\left(V_{A}\right) \phi^{j}+B_{m(A)-2} \phi^{(m(A)-2) / 2} \quad \text { if } m(A) \text { is even. }
\end{aligned}
$$

The Betti numbers are given by

$$
\left(\sum_{i=0}^{m\left(A_{1}\right)} x^{i}\right)\left(\sum_{j=0}^{m\left(A_{2}\right)} x^{j}\right)=\sum_{k=0}^{m(A)} B_{2 k}(A) x^{k},
$$

and

$$
\begin{array}{rlrl}
B_{2 j}\left(V_{A}\right) & =B_{2 j}(A) & & \text { for } 2 j<m(A)-2, \\
B_{2 j}\left(V_{A}\right) & =B_{2(m(A)-2)-2 j}^{(A)} & & \text { for } 2(m(A)-1)>2 j>m(A)-2, \\
B_{2(m(A)-1)}\left(V_{A}\right) & =B_{2(m(A))}\left(V_{A}\right)=1 .
\end{array}
$$

If $m(A) \leqq 1$, set $P_{A}(t)=\zeta\left(\bar{V}_{A}, t\right)=1$.

If $A \subset S_{1} \times S_{2}$ with $A_{1} \neq \Phi, A_{2} \neq \Phi$, then

$$
\zeta\left(\bar{V}_{A}, t\right)=\prod_{B_{1} \subset A_{1} ; B_{2} \subset A_{2}} \zeta\left(\bar{V}_{B}-\bar{V}_{B}^{\prime}, t\right)
$$

where $B=B_{1} \times B_{2}$ and $\bar{V}_{B}-\bar{V}_{B}^{\prime}$ has the obvious meaning.

This formula can be inverted by an analogue of the Möbius Inversion Formula to yield

$$
\zeta\left(\bar{V}_{A}-\bar{V}_{A}^{\prime}, t\right)=\prod_{B_{1} \subset A_{1} ; B_{2} \subset A_{2}} \zeta\left(\bar{V}_{B}, t\right)^{(-1)^{m(A)-m(B)}} .
$$


Now letting $A=S_{1} \times S_{2}$ and plugging (2) into (1), we see that

$$
\begin{array}{r}
(1-t)^{-(-\delta)^{n+m}} \chi_{F_{1}}^{-(-\delta)^{n+m+1}} \chi_{F_{2}}^{-(-\delta)^{n+m+1}} \chi_{\bar{F}}^{-(-\delta)^{n+m+2}(t)} \\
=\prod_{A_{1} \subset S_{1} ; A_{2} \subset S_{2}} \zeta\left(\bar{V}_{A}, q^{2} t\right)^{(-1)^{n+m-m(A)}} .
\end{array}
$$

Thus

$$
\chi_{F}(t)^{\delta^{n+m+2}}=(1-t)^{-\delta^{n+m}} \chi_{F_{1}}(t)^{\delta^{n+m+1}} \chi_{F_{2}}(t)^{\delta^{n+m+1}} \cdot \prod_{A_{1} \subset S_{1} ; A_{2} \in S_{2}} \zeta\left(\bar{V}_{A}, q^{2} t\right)^{(-1)^{1-m(A)}}
$$

But Ireland has shown [5] that

$$
\begin{aligned}
\chi_{F_{1}}^{\delta_{1}^{n+m+1}}(t) & =(1-t)^{\sum_{j=0}^{\infty}\left(\begin{array}{l}
n \\
j
\end{array}\right)\left(\begin{array}{l}
m \\
j
\end{array}\right) \phi^{j}} \cdot \prod_{A_{1} \subset S_{1} ; A_{2} \subset S_{2} ; m(A) \geqq 1} P_{A, 1}(q t), \\
\chi_{F_{2}}^{\delta^{n}+m+1}(t) & =(1-t)^{\sum_{j=0}^{\infty}\left(\begin{array}{l}
n \\
j
\end{array}\right)\left(\begin{array}{l}
m \\
j
\end{array}\right) \phi^{j}} \cdot \prod_{A_{1} \subset S_{1} ; A_{2} \subset S_{2}} P_{A, 2}(q t),
\end{aligned}
$$

where $P_{A, 1}$ and $P_{A, 2}$ are the rational functions corresponding to the divisors $\bar{f}(X, Y)$ and $\bar{g}(X, Y)$ respectively. Now substituting in our definitions of $P_{A}(t)$, we obtain after lengthy computation

THEOREM 1.

$$
\chi_{F}^{\delta^{n+m+2}}(t)=(1-t) \prod_{j=1}^{\infty}\left(1-q^{j} t\right)^{G(n, m, j)} \cdot \prod_{A_{1} \subset S_{1} ; A_{2} \in S_{2}} P_{A, 1}(q t) P_{A, 2}(q t) P_{A}\left(q^{2} t\right)
$$

where

$$
G(n, m, j)=\left(\begin{array}{c}
n \\
j
\end{array}\right)\left(\begin{array}{c}
m+1 \\
j
\end{array}\right)+\left(\begin{array}{c}
n+1 \\
j
\end{array}\right)\left(\begin{array}{c}
m \\
j
\end{array}\right)
$$

The vector space $W$. Consider the following graded ring $R=\sum_{k=0}^{\infty} R^{k} \cdot R^{0}=\Omega_{0}$, a finite extension field of $Q_{p}$ to be specified later $\left(T_{1} \subset \Omega_{0}\right) ; R^{k}=\Omega_{0}$-vector space generated by all monomials of the form

$$
Z_{1}^{a} Z_{2}^{b} X_{1}^{u} \cdots X_{n+1}^{u_{n+1}} Y_{1}^{v_{1}} \cdots Y_{m+1}^{v_{m+1}}
$$

with $a+b=k, d_{1} a+d_{2} b=\sum_{i=1}^{n+1} u_{i}, e_{1} a+e_{2} b=\sum_{j=1}^{m+1} v_{j}$. Let $m=\sum_{k=1}^{\infty} R^{k}$, the unique homogeneous maximal ideal of $R$.

We will later investigate this graded ring in great detail but for the time being, we will assume that $R$ is a homogeneously Macauley ring, i.e. $R_{m}$ is a Macauley local ring of dimension $n+m+2$.

Consider the ideal

$$
\mathfrak{A}=\left(Z_{1} f(X, Y), Z_{2} g(X, Y), X_{1} \frac{\partial h}{\partial X_{1}}, \ldots, X_{n+1} \frac{\partial h}{\partial X_{n+1}}, Y_{1} \frac{\partial h}{\partial Y_{1}}, \ldots, Y_{m+1} \frac{\partial h}{\partial Y_{m+1}}\right),
$$

where $h(Z, X, Y)=Z_{1} f(X, Y)+Z_{2} g(X, Y)$, generated by these $n+m+4$ elements of degree 1 in $R$. We recall now our earlier assumption that all the varieties $V_{A}$ are nonsingular. We now assume additionally that both $V_{1}$ and $V_{2}$ are nonsingular where these are the divisors corresponding to $f(X, Y)$ and $g(X, Y)$, respectively. 
Proposition 1. $\mathfrak{A}$ is an m-primary ideal in $R$.

Proof. We first show that $\mathfrak{A}$ has no nontrivial zero in $P^{1} \times\left(P^{n} \times P^{m}\right)$. Suppose $(z, x, y)$ is a zero of $\mathfrak{A}$. If $z_{1}=0$, then $z_{2} \neq 0$ which implies

$$
g(x, y)=x_{1} \frac{\partial g}{\partial x_{1}}=\cdots=x_{n+1} \frac{\partial g}{\partial x_{n+1}}=y_{1} \frac{\partial g}{\partial y_{1}}=\cdots=y_{m+1} \frac{\partial g}{\partial Y_{m+1}}=0 .
$$

But this implies that $(x, y)$ is a singular point of $V_{2}$ which is a contradiction. Similarly, if $z_{2}=0$, then $z_{1} \neq 0$ and we contradict $V_{1}$ being nonsingular. Thus, we can assume $z_{1} \neq 0, z_{2} \neq 0$. Then $f(x, y)=g(x, y)=0$ so $(x, y)$ is a point of $V$. But then $x_{i} \partial f / \partial x_{i}=-\left(z_{2} / z_{1}\right) x_{i} \partial g / \partial x_{i}$ and $y_{j} \partial f / \partial y_{j}=-\left(z_{2} / z_{1}\right) y_{j} \partial g / \partial y_{j}$ so again $(x, y)$ is a singular point of $V$. Thus $\mathfrak{A}$ has no nontrivial zero in $P^{1} \times\left(P^{n} \times P^{m}\right)$. The desired result now follows as a simple application of the Nullstellensatz.

Let $g_{1}, \ldots, g_{n+m+2}$ be any subset of the given set of generators of $\mathfrak{A}$. By the Euler relations $\mathfrak{A}=\left(g_{1}, \ldots, g_{n+m+2}\right)$. Hence by our assumption on $R, g_{1}, \ldots$, $g_{n+m+2}$ is a system of parameters for $R$. By Nagata [6], $g_{1}, \ldots, g_{n+m+2}$ is an $R-$ sequence as well.

Let $\mathfrak{A}^{k}=\mathfrak{A} \cap R^{k}$ and let $W^{k} \subset R^{k}$ be defined by the relation $R^{k}=W^{k}[+] \mathfrak{A}^{k}$. (For the definition of $p$-adic direct sums, see Dwork [4].) Define $W=\sum_{k=0}^{\infty} W^{k}$. We wish to compute $\operatorname{dim}_{\Omega_{0}} W^{k}$.

Consider the Koszul complex generated over $R$ by $g_{1}, \ldots, g_{n+m+2}$ which is exact by Auslander-Buchsbaum [1]. Looking at the following piece of the complex:

$$
\rightarrow \cdots \rightarrow\left(R^{k-j}\right)^{(n+m+2)} \rightarrow \cdots \rightarrow\left(R^{k-2}\right)^{(n+m+2)} \rightarrow\left(R^{k-1}\right)^{n+m+2} \rightarrow R^{k} \rightarrow W^{k} \rightarrow 0,
$$

we see

$$
\operatorname{dim} W^{k}=\sum_{j=0}^{\infty}(-1)^{j}\left(\begin{array}{c}
n+m+2 \\
j
\end{array}\right) \operatorname{dim} R^{k-j} .
$$

After lengthy computation we obtain

THEOREM 2.

$$
\operatorname{dim} W=\sum_{w_{1}+w_{2}=n ; v_{1}+v_{2}=m}\left(\begin{array}{c}
w_{1}+v_{1} \\
w_{1}
\end{array}\right)\left(\begin{array}{c}
w_{2}+v_{2} \\
w_{2}
\end{array}\right) d_{1}^{w_{1}} d_{2}^{w_{2}} e_{1}^{v} e_{2}^{v} .
$$

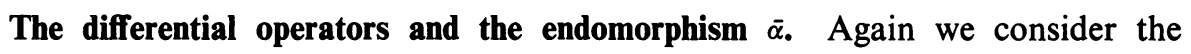
$\boldsymbol{m}$-primary ideal $\mathfrak{A}$ generated by homogeneous elements of degree one in $R$ and select a subset $g_{1}, \ldots, g_{n+m+2}$ of the given generating set of $\mathfrak{A}$. Then $\mathfrak{A}=$ $\left(g_{1}, \ldots, g_{n+m+2}\right)$, and $g_{1}, \ldots, g_{n+m+2}$ is an $R$-sequence.

Now consider $\Omega_{0}\{Z, X, Y\}=\Omega_{0}\left\{Z_{1}, Z_{2}, X_{1}, \ldots, X_{n+1}, Y_{1}, \ldots, Y_{m+1}\right\}$, the power series ring over $\Omega_{0}$. Let

$$
\begin{aligned}
L= & \left\{\xi=\sum A_{a, b, u, v} Z_{1}^{a} Z_{2}^{b} X^{u} Y^{v} \mid d_{1} a+d_{2} b=\sum u_{i}, e_{1} a+e_{2} b=\sum v_{j}\right\} \\
& \subset \Omega_{0}\{Z, X, Y\} .
\end{aligned}
$$


If $\xi \in L$, write $\xi=\sum_{k=0}^{\infty} \alpha_{k}(Z, X, Y)$ where $\alpha_{k}(Z, X, Y)$ is the sum of those terms of $\xi$ for which $a+b=k$. If $\alpha_{k} \neq 0$ and $\alpha_{i}=0, i<k$, then define $\nu(\xi)=k$.

Let $L(b, c)=\left\{\xi=\sum_{k=0}^{\infty} \alpha_{k}(Z, X, Y) \in L \mid\right.$ ord $A_{k} \geqq b k+c$ for any nonzero coefficient $A_{k}$ appearing in $\left.\alpha_{k}(Z, X, Y)\right\}$.

Let $L(b)=\bigcup_{c \in R} L(b, c)$. If $\xi \in L(b, c)$, then $\xi$ converges for ord $Z_{1}>-b$, ord $Z_{2}$ $>-b$, ord $X_{i}>0$, ord $Y_{j}>0$.

Choose an integer $\pi \in \Omega_{0}$ such that ord $\pi=1 / p-1$. Suppose $H \in \Omega_{0}\{Z, X, Y\}$ has the following properties:

(i) $H \in L(1 / p-1)$.

(ii) If we write $H=\sum_{k=0}^{\infty} \alpha_{k}(Z, X, Y)$, then $\alpha_{0}=0$ and $\alpha_{1}(Z, X, Y)=\pi\left(Z_{1} f+Z_{2} g\right)$.

$$
\begin{aligned}
E_{j} H & =X_{j}\left(\partial H / \partial X_{j}\right) \in L(p / p-1,-1), & & j=1, \ldots, n+1, \\
E_{n+1+j} H & =Y_{j}\left(\partial H / \partial Y_{j}\right) \in L(p / p-1,-1), & & j=1, \ldots, m+1, \\
E_{n+m+2+j} H & =Z_{j}\left(\partial H / \partial Z_{j}\right) \in L(p / p-1,-1), & & j=1,2 .
\end{aligned}
$$

We introduce the differential operators $D_{j}(\xi)=E_{j}(\xi)+\xi \cdot E_{j} H$, for $j=1, \ldots$, $n+m+4$. We have immediately the following relations:

(3)

$$
\begin{array}{r}
\sum_{i=1}^{n+1} D_{i}=d_{1} D_{n+m+3}+d_{2} D_{n+m+4}, \\
\sum_{j=1}^{m+1} D_{n+1+j}=e_{1} D_{n+m+3}+e_{2} D_{n+m+4} .
\end{array}
$$

Furthermore, $D_{i} \circ D_{j}=D_{j} \circ D_{i}$ and $D_{i}: L(b) \rightarrow L(b)$ for $b \leqq p / p-1$.

Following the proofs of [4, Lemmas 3.4-3.11], we obtain

THEOREM 3. For $1 / p-1<b \leqq p / p-1, L(b)=W \oplus \sum_{i=1}^{n+m+2} D_{i} L(b)$.

Again following [4, Theorem 3.1 and Corollary], we have

Proposition 2. The Koszul complex

$$
\begin{aligned}
\cdots \rightarrow L(b)^{(n+m+2)} \rightarrow \cdots \rightarrow L(b)^{(n+m+2)} \rightarrow L(b)^{n+m+2} & \rightarrow L(b) \\
& \rightarrow L(b) / \sum_{i=1}^{n+m+2} D_{i} L(b) \rightarrow 0,
\end{aligned}
$$

generated over $L(b)$ by the operators $D_{1}, \ldots, D_{n+m+2}$ is acyclic.

Proposition 3. $\operatorname{det}(I-t \bar{\alpha})=\chi_{F}^{\delta n+m+2}(t)$.

Proof. Since $\alpha \circ D_{i}=q D_{i} \circ \alpha$, we have the following exact commutative diagram.

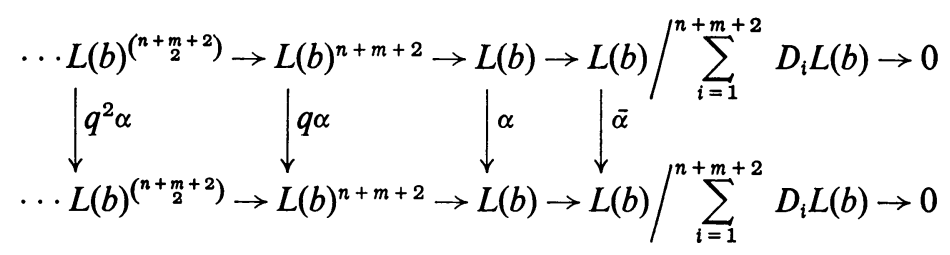

The result follows now from [7, Proposition 9]. 
Finally [4, Theorem 4.3] yields

THEOREM 4. $\bar{\alpha}$ is nonsingular for $b=p / p-1$.

The degree of $P(t)$ as a rational function and the Betti numbers. The following formula, due to Ireland, yields the Euler characteristic of $V$. Consider the expression

$$
\frac{(1+u)^{n+1}(1+v)^{m+1}}{\left(1+d_{1} u+e_{1} v\right)\left(1+d_{2} u+e_{2} v\right)}=\sum c_{i, j} u^{i} v^{j}
$$

Then

$$
\chi(V)=\sum_{j=0}^{2(m+n-2)}(-1)^{j} B_{j}(V)=d_{1} d_{2} c_{n-2, m}+\left(d_{1} e_{2}+d_{2} e_{1}\right) c_{n-1, m-1}+e_{1} e_{2} c_{n, m-2} .
$$

After a lengthy computation involving the formula of Theorem 1 and the Möbius Inversion Principle, we arrive at

THEOREM 5. The degree as a rational function of $P(t)$ is $B_{n+m-2}(V)$ when $n+m-2$ is odd and $B_{n+m-2}(V)+B_{n+m-2}\left(P^{n} \times P^{m}\right)$ when $n+m-2$ is even.

The graded ring $R$. We now return to the graded ring defined earlier to verify the assertion made there, namely,

THEOREM 6. $R$ is a homogeneously Macauley ring of dimension $n+m+2$.

It should be pointed out that the corresponding result in the case of a hypersurface is a triviality. It is not until one considers complete intersections that this problem occurs and it becomes even more complicated in the biprojective situation we are considering. The theorem follows as we will show from a result of Chow [2] and some technical lemmas developed to permit an iterative construction of $R$. We begin with a summary of the terminology and results of Chow.

Proposition 4. Let $A$ be a local ring of dimension d with maximal ideal $m$. Then the following statements are equivalent:

(i) There exists a system of parameters for $A$ which is an A-sequence.

(ii) Every system of parameters for $A$ is an A-sequence.

(iii) Any ideal in $A$ generated by $r$ elements and of rank $r$ is unmixed.

Definition. A local ring satisfying any (hence all) of the above conditions is called Macauley.

Now let $A=\sum_{i=0}^{\infty} A_{i}$ be a graded Noetherian ring with $A_{0}$ a field. Let $\boldsymbol{m}=\sum_{i=1}^{\infty} A_{i}$ be the unique homogeneous maximal ideal of $A$.

Definition. We say that $A$ satisfies $H$-unmixedness if every homogeneous ideal in $A$ generated by $r$ elements and of rank $r$ is unmixed.

Proposition 5. A satisfies H-unmixedness if and only if $A_{m}$ is Macauley.

Definition. A graded Noetherian ring $A$ with a unique homogeneous maximal ideal $\boldsymbol{m}$ will be called an $H$-local ring. We define $\operatorname{dim} A=\operatorname{rank} \boldsymbol{m}$. 
Definition: Let $A$ be an $H$-local ring of dimension $d$. Then a set of homogeneous elements $x_{1}, \ldots, x_{d} \in A$ is said to be an $H$-system of parameters if $A\left(x_{1}, \ldots, x_{d}\right)$ is an $m$-primary ideal.

Definition. An $H$-local ring is said to be $H$-Macauley if it contains an $H$ system of parameters which is an $A$-sequence.

Proposition 6. Let $A$ be an $H$-local ring with $A_{0}$ a field. Then $A$ satisfies $H$ unmixedness if and only if $A$ is $H$-Macauley.

Definition. Let $A=\sum_{i=0}^{\infty} A_{i}, B=\sum_{j=0}^{\infty} B_{j}$ be $H$-local rings with $A_{0}=B_{0}=K$, a field. For any pair of positive integers $(d, e)$, we define the Segre product of $A$ and $B$ of $\operatorname{order}(d, e)$ to be the $H$-local ring

$$
C=\sum_{k=0}^{\infty} C_{k} \text { where } C_{k}=A_{k d} \otimes_{K} B_{k e} .
$$

We next pose the following question: If $A$ and $B$ are $H$-Macauley, under what conditions is the Segre product of $A$ and $B$ of order $(d, e)$ again $H$-Macauley? In order to answer this question we must introduce the notion of " $d$-properness".

From now on we will mean by an $H$-system of parameters for an $H$-local ring $A$ a system of parameters consisting of homogeneous elements all of the same degree, called the degree of the system.

Definition. Let $x_{1}, \ldots, x_{m}$ be an $H$-system of parameters for an $H$-local ring $A$ ( $\operatorname{dim} A=m$ ) of degree $f d$. We shall say that this system of parameters has the $d$ exponent $\varepsilon$ if $\varepsilon$ is the least positive integer with the property that every homogeneous element of $A$ of degree $k d$ with $k \geqq \varepsilon f$ is contained in $A\left(x_{1}, \ldots, x_{m}\right)$.

Proposition 7. If $A$ is $H$-Macauley, then every $H$-system of parameters of degree fd has the same d-exponent.

Proposition 8. If $A$ is $H$-Macauley and if one (hence every) $H$-system of parameters of degree fd has d-exponent less than or equal to $m$, then the same is true for every $H$-system of parameters of degree $f^{\prime} d$ for any $f^{\prime}$.

Combining the two preceding propositions, we see that the property of having $d$-exponent less than or equal to the dimension of the ring is independent of the choice of an $H$-system of parameters in an $H$-Macauley ring. Thus we make the following definition.

Definition. An $H$-Macauley ring $A$ of dimension $m$ is said to be $d$-proper (or just proper for $d=1$ ) if there exists an $H$-system of parameters in $A$ of $d$-exponent less than or equal to $m$.

We note that $d$-proper implies $d^{\prime}$-proper for any $d^{\prime}$ a multiple of $d$. In particular, proper implies $d$-proper for all $d$.

THEOREM 7. Let $A$ and $B$ be $H$-Macauley rings of dimensions $m$ and $n$, respectively, $A_{0}=B_{0}=K$, a field. Let $C$ denote the Segre product of $A$ and $B$ of order $(d, e)$. Then: 
(i) If $A$ is d-proper (unless $n=1$ ) and $B$ is e-proper (unless $m=1$ ), then $C$ is $H$ Macauley of dimension $m+n-1$.

(ii) If $A$ and $B$ contain nonzero homogeneous elements of all degrees which are multiples of $d$ and e, respectively, and $C$ is $H$-Macauley, then $A$ is $d$-proper (unless $n=1$ ) and $B$ is e-proper (unless $m=1$ ).

(iii) If $A$ is d-proper and $B$ is e-proper, then $C$ is proper.

This concludes the summary of [2] and we now proceed to the proof of Theorem 6.

Let $A=\Omega_{0}\left[Z_{1}, Z_{2}\right]$ graded by $\operatorname{deg} Z_{1}=d_{1}, \operatorname{deg} Z_{2}=d_{2} ; B=\Omega_{0}\left[X_{1}, \ldots, X_{n+1}\right]$ graded by $\operatorname{deg} X_{i}=1 ; T=\Omega_{0}\left[Y_{1}, \ldots, Y_{m+1}\right]$ graded by $\operatorname{deg} Y_{j}=1$. It can be seen that each of these is a proper $H$-Macauley ring of dimension $2, n+1, m+1$, respectively. Let $S^{\prime}=\sum_{k=0}^{\infty} S_{k}^{\prime}$ be the Segre product of order $(1,1)$ of $A$ and $B$ which. by the previous theorem, is a proper $H$-Macauley ring of dimension $n+2 . S_{0}^{\prime}=\Omega_{0}$ and $S_{k}^{\prime}=$ the $\Omega_{0}$-vector space generated by

$$
\left\{Z_{1}^{a} Z_{2}^{b} X^{u} \mid \sum_{i=1}^{n+1} u_{i}=d_{1} a+d_{2} b=k\right\}
$$

Let $\boldsymbol{m}^{\prime}=\sum_{k=1}^{\infty} S_{k}^{\prime}$. Now consider the graded ring $S=\sum_{k=0}^{\infty} S_{k}$ where $S_{0}=\Omega_{0}$ and $S_{k}=$ the $\Omega_{0}$-vector space generated by

$$
\left\{Z_{1}^{a} Z_{2}^{b} X^{u} \mid \sum_{i=1}^{n+1} u_{i}=d_{1} a+d_{2} b, k=e_{1} a+e_{2} b\right\}
$$

Let $\boldsymbol{m}=\sum_{k=1}^{\infty} S_{k}$. Since $d_{1} a+d_{2} b>0$ if and only if $e_{1} a+e_{2} b>0$, we see that $S_{\boldsymbol{m}}=S_{\boldsymbol{m}^{\prime}}^{\prime}$ which is Macauley by Proposition 7 and Proposition 8. Hence $S$ is $H$-Macauley by the same two propositions. Let us assume for the moment that $S$ is also proper. Then denote by $R^{\prime}=\sum_{k=0}^{\infty} R_{k}^{\prime}$ the Segre product of order $(1,1)$ of $S$ and $T$; we see that $R^{\prime}$ is a proper $H$-Macauley ring of dimension $n+m+2 . R_{0}^{\prime}=\Omega_{0}, R_{k}^{\prime}=$ the $\Omega_{0}$-vector space generated by

$$
\left\{Z_{1}^{a} Z_{2}^{b} X^{u} Y^{v} \mid \sum_{i=1}^{n+1} u_{i}=d_{1} a+d_{2} b, \sum_{j=1}^{m+1} v_{j}=e_{1} a+e_{2} b=k\right\}
$$

We can now obtain our ring $R$ from $R^{\prime}$ in the same way that $S$ was obtained from $S^{\prime}$, yielding $R$ as an $H$-Macauley ring of dimension $n+m+2$. It remains only to show that the ring $S$ is proper.

Suppose that $d_{1} e_{2} \geqq d_{2} e_{1}$. Consider the following set of homogeneous elements of $S$ :

$$
\begin{gathered}
w_{1}=Z_{1}^{e_{2}} X_{1}^{d_{1} e_{2}}, \quad w_{i}=Z_{1}^{e_{2}} X_{i}^{d_{1} e_{2}}-Z_{2}^{e_{1}} X_{i-1}^{d_{2} e_{1}}, \quad i=2, \ldots, n+1, \\
w_{n+2}^{w_{2}}=Z_{2}^{e_{1}} X_{n+1}^{d_{2} e_{1}} .
\end{gathered}
$$

We note that the degree of each of these elements is $e_{1} e_{2}$. Let $\mathfrak{A}=S\left(w_{1}, \ldots, w_{n+2}\right)$. In order to show that $S$ is proper it suffices to prove that $\mathfrak{A} \supset \boldsymbol{m}^{(n+2) e_{1} e_{2}}$. This would in particular prove that $w_{1}, \ldots, w_{n+2}$ form an $H$-system of parameters for $S$. 
Lemma. Let $\alpha=Z_{1}^{a} Z_{2}^{b} X^{u} \in S$. Suppose that for some $1 \leqq i<j \leqq n+1$, we have (i) $u_{i} \geqq d_{2} e_{1}$ and (ii) $b-(j-i) e_{1} \geqq 0$. Then $\alpha \equiv \beta(\mathfrak{A})$ where $\beta=Z_{1}^{a+(j-i) e_{2}} Z_{2}^{b-(j-1) e_{1}} X^{u^{\prime}}$ and $u_{i}^{\prime}=u_{i}-d_{2} e_{1}, u_{j}^{\prime}=u_{j}+d_{1} e_{2}, u_{k}^{\prime}=u_{k}$ for $1 \leqq k<i, j<k \leqq n+1, u_{k}^{\prime}=u_{k}+\left(d_{1} e_{2}-d_{2} e_{1}\right)$ for $i<k<j$.

Proof. Successively reduce $\alpha$ modulo $w_{i+1}, w_{i+2}, \ldots, w_{j}$ in order to obtain $\beta$.

Proposition 9. $S$ is proper, i.e. $\mathfrak{A} \supset \boldsymbol{m}^{(n+2) e_{1} e_{2}}$.

Proof. Let $\alpha=Z_{1}^{a} Z_{2}^{b} X^{u} \in m^{(n+2) e_{1} e_{2}}$. Thus $\sum_{i=1}^{n+1} u_{i}=d_{1} a+d_{2} b$ and $e_{1} a+e_{2} b$ $\geqq(n+2) e_{1} e_{2}$. Consider the statement $P(k)$ : If $(k-1) e_{2} \leqq a<k e_{2}$, then $\alpha \in \mathfrak{A}$. We will verify $P(k)$ for all $k$, thereby showing $\alpha \in \mathfrak{A}$.

Suppose $0 \leqq a<e_{2}$. Then $b>(n+1) e_{1}$. Hence $\sum_{i=1}^{n+1} u_{i}=d_{1} a+d_{2} b>(n+1) d_{2} e_{1}$ so some $u_{i}>d_{2} e_{1}$. If $u_{n+1}>d_{2} e_{1}$, then $w_{n+2} \mid \alpha$ and we are done. So we can assume $i<n+1$. Now by the preceding lemma,

$$
\alpha \equiv \beta=Z_{1}^{a+(n+1-i) e_{2}} Z_{2}^{b-(n+1-i) e_{1}} X_{1}^{u_{1}^{\prime}} \cdots X_{n}^{u_{n}^{\prime}} X_{n+1}^{u_{n}+1+d_{1} e_{2}}(\mathfrak{A}) .
$$

But $b-(n+1-i) e_{1}>e_{1}$ and $u_{n+1}+d_{1} e_{2} \geqq d_{2} e_{1}$ so that $w_{n+2} \mid \beta$. Hence $\alpha \in \mathfrak{A}$, verifying $P(1)$.

Now suppose $P(1), P(2), \ldots, P(m)$ to be true and assume $m e_{2} \leqq a<(m+1) e_{2}$. Then $(m+1) e_{1} e_{2}+e_{2} b>e_{1} a+e_{2} b \geqq(n+2) e_{1} e_{2}$ gives $b>(n-m+1) e_{1}$. We can also assume $u_{i}<d_{1} e_{2}$ for $i=1, \ldots, n+1$. For if $u_{1} \geqq d_{1} e_{2}$, then $w_{1} \mid \alpha$ and we are done. If $u_{i} \geqq d_{1} e_{2}$ for some $i=2, \ldots, n+1$, then $\alpha \equiv Z_{1}^{a-e_{2}} Z_{2}^{b+e_{1}} X^{u^{\prime}} \bmod w_{i}$ where $u_{i}^{\prime}=u_{i}$ $-d_{1} e_{2}, u_{i-1}^{\prime}=u_{i-1}+d_{2} e_{1}, u_{j}^{\prime}=u_{j}$ for $j \neq i-1, i$. But $Z_{1}^{a-e_{2}} Z_{2}^{b-e_{1}} X^{u^{\prime}} \in \mathfrak{A}$ by $P(m)$.

Next we can assume $m \leqq n$. For if $m \geqq n+1$, then since $u_{1}<d_{1} e_{2}$, we have

$$
\sum_{i=2}^{n+1} u_{i}>d_{1} a+d_{2} b-d_{1} e_{2} \geqq(m-1) d_{1} e_{2} \geqq n d_{1} e_{2}
$$

in which case some $u_{i}>d_{1} e_{2}$ which we have shown cannot happen. Now

$$
\sum_{i=2}^{n+1} u_{i}>d_{1} a+d_{2} b-d_{1} e_{2} \geqq(m-1) d_{1} e_{2}+(n-m+1) d_{2} e_{1}
$$

and since $\sum_{i=2}^{m} u_{i}<(m-1) d_{1} e_{2}$, we get $\sum_{i=m+1}^{n+1} u_{i}>(n-m+1) d_{2} e_{1}$. But this implies some $u_{j}>d_{2} e_{1}$ for $m+1 \leqq j \leqq n+1$. Now applying the preceding lemma, we see that

$$
\alpha \equiv \beta=Z_{1}^{a+(n+1-j) e_{2}} Z_{2}^{b-(n+1-j) e_{1}} X^{u^{\prime}}
$$

where $u_{n+1}^{\prime}=u_{n+1}+d_{1} e_{2} \geqq d_{2} e_{1}$. Since $b-(n+1-j) e_{1} \geqq b-(n-m) e_{1}>e_{1}$, we have that $w_{n+2} \mid \beta$ so $\alpha \in \mathfrak{A}$. We have now shown that $P(m+1)$ is also valid and so $P(k)$ is true for all $k$, completing the proof of the proposition.

Thus we have shown that $S$ is proper for the case $d_{1} e_{2} \geqq d_{2} e_{1}$. But the case $d_{2} e_{1}$ $\geqq d_{1} e_{2}$ can clearly be handled in a similar manner, thus completing the proof of Theorem 6. 


\section{REFERENCES}

1. M. Auslander and D. A. Buchsbaum, Codimension and multiplicity, Ann. of Math. 68 (1958), 625-657.

2. W.-L. Chow, On unmixedness theorem, Amer. J. Math. 86 (1964), 799-822.

3. B. M. Dwork, On the rationality of the zeta function of an algebraic variety, Amer. J. Math. 82 (1960), 631-648.

4. - On the zeta function of a hypersurface, Publ. Math. Inst. Hautes Etudes Sci., 12, Paris, 1962.

5. K. F. Ireland, On the zeta function of an algebraic variety, Ph.D. Dissertation, Johns Hopkins Univ., Baltimore, Md., 1964.

6. M. Nagata, Local rings, Interscience, New York, 1962.

7. J.-P. Serre, Endomorphismes complètement continus des espaces de Banach p-adiques, Publ. Math. Inst. Hautes Etudes Sci., 12, Paris, 1962.

8. A. Weil, Numbers of solutions of equations in finite fields, Bull. Amer. Math. Soc. 55 (1949), 497-508.

\section{NORTHEASTERN UNIVERSITY,}

Boston, Massachusetts 